\title{
Construção e validação do instrumento "Inventário de problemas éticos na atenção primária em saúde"
}

José Roque Junges ${ }^{1}$, Elma Lourdes Campos Pavone Zoboli ${ }^{2}$, Marcos Paschoal Patussi ${ }^{3}$, Rafaela Schaefer ${ }^{4}$, Carlise Rigon Della Nora ${ }^{5}$

\section{Resumo}

Identificar conflitos éticos contribui para melhorar a qualidade da assistência. $O$ estudo objetivou verificar a validade de construto e consistência interna do "Inventário de problemas éticos na atenção primária em saúde". O instrumento, questionário estruturado e fechado, é fruto de uma década de pesquisas sobre bioética na atenção básica. $O$ artigo enfoca a última etapa da validação. No início dessa fase, o instrumento continha 41 itens. Foi aplicado a 237 profissionais de 12 unidades de saúde do Grupo Hospitalar Conceição (Porto Alegre/RS). Após análise fatorial exploratória dos escores, obtiveram-se seis dimensões centrais com elevado grau de confiabilidade e consistência (KMO=0,831; Bartlett $p<0,001 ; \alpha$ geral 0,876 ), explicando $61,4 \%$ da variância. Ao final, o instrumento validado ficou com seis fatores e 24 itens. $O$ instrumento poderá contribuir para pesquisas em bioética, com estudos quantitativos em grandes amostras, bem como propiciar o reconhecimento dos problemas éticos mais comuns na atenção básica.

Palavras-chave: Estudos de validação. Ética. Bioética. Atenção primária à saúde. Resolução de problemas.

\section{Resumen}

\section{La construcción y validación del "Inventario de problemas éticos en la atención primaria de la salud"}

Identificar los conflictos éticos contribuye para mejorar la calidad de la atención. Este estudio tuvo como objetivo evaluar la validez de constructo y la consistencia interna del Inventario de Problemas Éticos en la Atención Primaria de la Salud. El instrumento, un cuestionario estructurado y cerrado, es resultado de una década de investigaciones sobre bioética en la atención primaria. El artículo enfoca la última etapa de validación. En el inicio de esta etapa, el instrumento contenía 41 elementos. Se lo ha aplicado a 237 profesionales de 12 centros de salud del Grupo Hospitalar Conceição (Porto Alegre/RS). Tras el análisis factorial exploratorio de las puntuaciones, se encontró seis dimensiones centrales con alto grado de fiabilidad y consistencia (KMO = 0,831 , Bartlett $p<0,001$, alpha 0,876), explicando el $61,4 \%$ de la varianza. La versión validada del instrumento quedó con 6 factores y 24 ítems. El instrumento contribuirá para las investigaciones en bioética, pues posibilita estudios cuantitativos en grandes muestras para llevar a reconocerse los problemas éticos más frecuentes en atención primaria.

Palabras-clave: Estudios de validación. Ética. Bioética. Atención primaria de salud. Solución de problemas.

\begin{abstract}
Construction and validation of the instrument "Inventory of ethical problems in primary health care"

Identifying ethical conflicts contributes to improve healthcare quality. This study aimed to evaluate content validity and verify internal consistency of the Inventory of Ethical Problems in Primary Health Care. The instrument - a close and structured questionnaire - is a result of decades of researches on bioethics in primary health care. The article focuses on the last stage of validation. At the beginning of this stage the instrument had 41 items. It was applied to 237 professionals of 12 primary care centers at Grupo Hospitalar Conceição (Porto Alegre/RS). After the exploratory factor analysis, it resulted in six dimensions with high degree of reliability and consistency (KMO $=0.831$, Bartlett $p<0.001$; alpha 0.876 ), which explained $61.4 \%$ of variance. At the end, the validated version of the instrument had 6 factors and 24 items. The instrument may contribute to researches in bioethics through the development of studies to recognize the most common ethical problems in primary care and to explore the influence of different contexts on ethical problems.
\end{abstract}

Key words: Validation studies. Ethics. Bioethics. Primary health care. Problem solving.

Aprovação CEP/Grupo Hospitalar Conceição 09-156

1. Doutor roquejunges@hotmail.com - Unisinos, São Leopoldo/RS, Brasil 2. Livre docente elma@usp.br - Universidade de São Paulo, São Paulo/SP, Brasil 3. Doutor mppatussi@unisinos.br - Unisinos, São Leopoldo/RS, Brasil 4. Doutoranda rafaelaschaefer@hotmail.com - Universidade Católica Portuguesa, Porto, Portugal 5. Doutoranda carliserdn@yahoo.com.br - Universidade Católica Portuguesa, Porto, Portugal.

Declaram não haver conflito de interesse. 
Investigações sobre os desafios éticos nos serviços de atenção à saúde podem levar os profissionais a refletir, deliberar e avaliar atitudes éticas nas práticas de saúde ${ }^{1}$. Os problemas éticos são desafios que exigem deliberação pela melhor solução. Entendidos como desafios, não podem ser resolvidos por meio de receitas prontas, mas exigem permanente criatividade, porque requerem respostas de longo alcance, que ultrapassem a solução de um caso particular $^{2}$. No Sistema Único de Saúde (SUS), a Estratégia de Saúde da Família (ESF) vem reorganizando a Atenção Primária à Saúde (APS) por meio da efetivação dos princípios da longitudinalidade e da integralidade. Tal fato reforça a necessidade de compromissos éticos das equipes, que têm de exercer nova prática marcada pela humanização, cuidado e cidadania ${ }^{1}$.

Nessa perspectiva, desde 2000 temos estudado a temática da ética na APS. O primeiro estudo, entre 2000 e 2003, desenvolveu uma pesquisa qualitativa com enfermeiros e médicos de equipes de saúde da família do município de São Paulo, objetivando reconhecer um perfil das questões éticas nessa nova forma de organização da APS no SUS. Como resultado, obteve-se uma lista de 41 problemas ${ }^{1}$, que originaram a primeira versão do Inventário de problemas éticos na atenção primária (IPE-APS).

Entre 2004 e 2005, desenvolveu-se um segundo estudo para reconhecer se os problemas apontados no primeiro se repetiriam na vivência de outras equipes de saúde da família e se ainda haveria algo a acrescentar. $O$ estudo mostrou que a primeira versão do IPE-APS era de fácil compreensão e preenchimento, contendo uma lista de problemas éticos da prática na APS suficientemente abrangente, não sendo necessário incluir novos exemplos ${ }^{3}$.

De 2005 a 2009, no município de São Paulo, outros dois estudos qualitativos investigaram as situações eticamente significativas vividas por enfermeiros e médicos em serviços de APS não reorganizados pela ESF. Uma dessas pesquisas investigou as unidades básicas de saúde (UBS) tradicionais e a outra enfocou os centros de saúde escola ${ }^{4}$. Os resultados comprovaram a abrangência da lista de problemas que compunha a primeira versão do IPE-APS. Um terceiro estudo desse mesmo período validou a sensibilidade do instrumento. Para essa etapa de validação foi desenvolvido um estudo com expertos da área de ética e bioética. $O$ instrumento mostrouse sensível, permitindo captar a variação de visão segundo o grupo em que era aplicado: profissionais da APS e especialistas em ética e bioética ${ }^{5}$.

Com esses estudos, viu-se que a primeira versão do IPE-APS se mostrava abrangente, sen- sível, bastante compreensível e de fácil aplicação. $\mathrm{Na}$ continuidade da validação, era preciso avaliar o construto do instrumento. Para validar a clareza, a facilidade de leitura e a confiabilidade do construto desenvolveu-se, entre 2006 e 2008, um estudo metodológico ${ }^{6}$. A validação da clareza e confiabilidade deu-se por meio de um painel de especialistas em saúde da família, com uso da técnica Delphi.

Cada item do IPE-APS teve seu enunciado descrito segundo a situação narrada pelos entrevistados do estudo, cujos resultados deram origem à lista de problemas constantes do inventário. Para cada item do IPE-APS solicitava-se aos especialistas do painel que avaliassem a congruência entre o enunciado do problema ético e a descrição da situação que o gerou. Havia espaço para que os especialistas sugerissem nova redação para o enunciado, se fosse o caso. O ponto de corte da congruência era $80 \%$, sendo mantida a redação dos enunciados com valor igual ou superior $a$ isso ${ }^{6}$.

Para a validação da facilidade de leitura usouse o Índice de Facilidade de Leitura de Flesch-Kincaid (ILFK), disponível no Microsoft Windows Word. A redação final dos enunciados dos problemas éticos resultante da consulta ao painel de especialistas foi ajustada para uma facilidade de leitura compatível com uma escolaridade aproximada ao $7^{\circ}$ ano (antiga $6^{a}$ série) do ensino fundamental (leitura razoavelmente fácil). A redação ajustada para cada item foi revista por uma professora de língua portuguesa, para verificar a adequação e correção da escrita. Com esse estudo metodológico fechou-se, em 2008, a $2^{\mathrm{a}}$ versão do IPE-APS ${ }^{6}$.

Até essa segunda versão o IPE-APS vinha sendo validado na vertente qualitativa. Sua escala para registro da ocorrência dos problemas éticos era nominal e não numérica. A partir de 2009 , usando a segunda versão do IPE-APS, iniciou-se a validação por meio de técnicas quantitativas, para viabilizar a incorporação de uma escala do tipo Likert no instrumento. Com isso, seria possível seu uso em estudos quantitativos, com amostras maiores. As pesquisas objetivaram verificar a validade de construto e a consistência interna do IPE-APS. O presente artigo descreve os resultados da última dessas pesquisas de validação do IPE-APS.

\section{Método}

Estudo transversal, realizado no Rio Grande do Sul, com profissionais de 12 unidades de saúde do Grupo Hospitalar Conceição (GHC), instituição pú- 
blica federal vinculada ao Ministério da Saúde, referência no atendimento do SUS. O Grupo conta com uma equipe de 7.913 profissionais e abrange quatro hospitais (Conceição, Criança Conceição, Cristo Redentor e Fêmina), 12 postos de saúde do Serviço de Saúde Comunitária, três centros de atenção psicossocial (Caps) e o Centro de Educação Tecnológica e Pesquisa em Saúde, a Escola GHC. É nacionalmente reconhecido como a maior rede pública de hospitais do Sul do Brasil, com $100 \%$ de atendimento SUS ${ }^{7}$.

O trabalho de campo teve início em julho de 2011 com um estudo piloto, realizado na unidade de saúde Conceição, que incluiu cerca de 60 profissionais. O propósito era conhecer a realidade in loco para organizar o trabalho de campo e, também, observar a compreensibilidade do instrumento pelos profissionais durante o seu preenchimento, vez que só havia sido utilizado em estudos no município de São Paulo.

A coleta de dados ocorreu entre agosto e dezembro de 2011, aproveitando-se as reuniões semanais das equipes. Os profissionais eram convidados a participar de forma voluntária, formando uma amostra por conveniência. Após os esclarecimentos sobre a pesquisa e a liberdade de participação, todos os presentes à reunião recebiam o IPE-APS, com as instruções para seu preenchimento. Ao final, recolhiam-se todos os instrumentos distribuídos, estivessem ou não preenchidos. Como não havia identificação do respondente se preservava o anonimato dos incluídos no estudo e também dos que se recusaram a participar, já que a retirada dos instrumentos em branco não era feita no campo.

A amostra totalizou 237 profissionais. Para o procedimento de análise fatorial exploratória, autores mais rigorosos, como Dassa ${ }^{8}$, sustentam que o tamanho ideal da amostra é de, no mínimo, dez sujeitos por item do inventário ou um total de pelo menos 250 sujeitos. Outros autores, como Pestana e Gageiro ${ }^{9}$, propõem, no mínimo, cinco sujeitos por questão em inventários com mais de 15 itens, considerando-se um mínimo de 100 sujeitos. Frente a isso, consideramos a amostra do presente estudo suficiente para as análises propostas.

A versão do IPE-APS submetida à validação quantitativa continha os 41 itens da segunda versão da validação qualitativa, com problemas éticos em três âmbitos relacionais da atenção à saúde: usuário-profissional, equipes de saúde da família e sistema de saúde ${ }^{1}$. A cada item os respondentes podiam expressar sua visão sobre o tema numa escala que variava de zero a quatro. $O$ zero significava que o respondente não considerava problema ético a si- tuação enunciada no item. Caso contrário, tinha que assinalar com que frequência encontrava o problema em seu trabalho na APS: nunca (1); raramente (2); frequentemente (3); sempre (4).

Registraram-se, também, algumas características dos respondentes: socioeconômicas, especialmente a escolaridade; demográficas (sexo, cor ou raça e estado civil); fatores relacionados ao processo de trabalho (profissão, tempo de formação, tempo de trabalho na APS e tempo de trabalho na unidade de saúde atual).

O Epidata versão 3.1 foi utilizado para a digitação dos dados e o software Statistical Package for the Social Sciences (SPSS) versão 12.0, para a análise. Com o objetivo de avaliar a adequação dos dados à análise fatorial, usou-se o teste de KaiserMeyer-Olkin (KMO). Para a validação do constructo fez-se uso da análise de componentes principais e a rotação varimax. Para tanto, foram seguidos os passos propostos por Hair JF, Anderson RE, Tatham RL e Black WC ${ }^{10}$ : formulação do problema; construção da matriz de correlação; determinação do método de análise fatorial; determinação do número de fatores; rotação dos fatores; interpretação dos fatores; cálculo das cargas fatoriais ou escolha de variáveis substitutas, e determinação do ajuste do modelo.

Para encontrar a melhor solução em termos de números de fatores utilizaram-se como critérios: autovalor maior ou igual a 1, mínimo de três itens por fator, e coerência com a teoria que fundamentou a construção do instrumento em todos os estudos: a bioética deliberativa de Diego Gracia. Os critérios de remoção de itens foram: aumento de alpha após a remoção do item; item carregando vários fatores ao mesmo tempo, e remoção do item cujo conteúdo não fosse coerente com o construto. Para avaliar a consistência interna da escala total e sua subescala utilizou-se o alpha de Cronbach ${ }^{11}$.

A pesquisa foi aprovada pelo Comitê de Ética em Pesquisa do Grupo Hospitalar Conceição e todos os participantes assinaram o termo de consentimento livre e esclarecido.

\section{Resultados}

Dentre outros profissionais de saúde das equipes de saúde da família, a amostra de 237 profissionais incluía médicos (14\%), agentes comunitários de saúde (11\%), auxiliares e/ou técnicos em enfermagem (10\%) e enfermeiros (9\%). Predominou o sexo feminino (79\%), $82 \%$ se consideravam brancos, $50 \%$ eram solteiros e $60 \%$ apresentavam idade entre 21 e 40 anos. 
A análise dos dados considerou as respostas dos 237 profissionais participantes. Com o intuito de avaliar a estrutura geral dos dados, rodou-se a análise no SPSS de forma aberta, ou seja, sem delimitar previamente a quantidade de fatores, com rotação varimax. $O$ resultado dessa análise indicou a existência de 10 diferentes fatores, nos quais índice $\mathrm{KMO}=0,847$ e Bartlett $\mathrm{p}<0,001$. A variância explicada nesta solução era de 63,5\% e o alpha geral 0,921. Essas medidas indicaram que os resultados eram satisfatórios, ou seja, mostraram a adequação dos dados para se proceder ao modelo exploratório de análise fatorial.

Após a verificação da adequação dos dados, para a sequência da análise foram retirados os itens que, ao mesmo tempo, apresentavam dois ou mais fatores ainda que com baixa comunalidade. Retiraram-se, um a um, seis itens, o que aumentou a variância para 64,4\%.

Após a retirada desses itens, a análise gerava nove fatores. Três fatores tinham apenas dois itens, o que não é recomendável na validação, segundo autores como Tabachnick e Fidell ${ }^{11}$. Portanto, optou-se por retirar mais seis itens. Com isso, a explicação da variância diminuiu para $59,6 \%$. Não se esperava esse comportamento da variância com a remoção dos itens, mas, apesar disso, devido à necessidade de eliminar fatores que continham apenas dois itens, optou-se pela retirada.

Ao se analisar a consistência teórica, foram encontrados três itens cujos problemas éticos enunciados não faziam sentido nos fatores onde se alocaram. Também se optou por sua retirada. Obteve-se, assim, a explicação de $61,4 \%$ da variância em um modelo com seis fatores e 24 itens. $O$ índice $\mathrm{KMO}$ ficou em 0,831, Bartlett $p<0,001$ e alpha geral de 0,876 (tabela 1). Esse arranjo de itens e fatores foi considerado a melhor solução de análise, pois combinou importantes resultados estatísticos e agrupou os problemas éticos de forma apropriada do ponto de vista teórico ${ }^{11}$.

Os fatores podem ser tidos como categorias de análise ou, ainda, domínios de avaliação do IPE-APS. Eles agruparam os problemas éticos na APS por frequência e por suas características, os que mais acontecem e de que tipo são, com base em publicações sobre atenção básica ${ }^{12}:$ 1) Gestão da atenção primária; 2) Longitudinalidade; 3) Prática das equipes; 4) Perfil profissional; 5) Privacidade na atenção primária à saúde e; 6) Sigilo profissional (Tabela 1 - Anexo).

O domínio 'gestão da atenção primária' contém seis itens, com alpha de Cronbach igual a 0,854.
Agrupa problemas éticos referentes à: carência de condições para os atendimentos; falta de condições para a realização de visitas domiciliares; deficiências do serviço de retaguarda para a remoção de pacientes; dificuldades no sistema de referência e contrarreferência; retorno e confidencialidade dos resultados de exames laboratoriais e; excesso de famílias adscritas a cada equipe na ESF. Esse domínio do IPE-APS traz a questão dos aspectos diretamente relacionados à organização e ao funcionamento dos serviços como fonte de problemas éticos para os profissionais de saúde na ESF.

O fator 'longitudinalidade' contém quatro itens que tratam das questões relacionadas à: continuidade de tratamento; recusa em seguir a indicação médica; prescrição de medicamento que o usuário não terá dinheiro para comprar e; dificuldade de cumprir na prática as responsabilidades de cada profissional da equipe. Esse domínio do IPE-APS engloba os problemas éticos decorrentes da prolongada relação que se estabelece entre o profissional e o usuário. O alpha de Cronbach desse fator foi 0,70.

No fator 'prática das equipes' estão quatro itens que expressam problemas éticos decorrentes da fragmentação do trabalho das equipes e a dificuldade de exercer a prática interdisciplinar. Esse domínio trata da: falta de respeito entre os membros da equipe; falta de colaboração de uma equipe com a outra; profissionais que não apresentam perfil para trabalhar na ESF e; profissionais que atuam com falta de compromisso e envolvimento. O alpha de Cronbach desse fator foi 0,76.

Três itens formam o fator 'perfil profissional', com alpha de Cronbach de 0,72. São problemas éticos relativos ao perfil atitudinal do profissional na APS, reorganizada ou não pela ESF: pré-julgamento de usuários e familiares; falta de respeito e prescrições inadequadas.

O quinto fator, 'privacidade na atenção primária à saúde', compreende três itens, que trazem aspectos próprios deste tema ético no contexto da APS, onde os domicílios e a comunidade são extensões do consultório. O fator teve alpha de Cronbach igual a 0,70.

O fator 'sigilo profissional' teve alpha de Cronbach igual a 0,64 e agrupou quatro itens relativos aos problemas éticos nas informações sobre a saúde dos usuários e o compartilhamento destas entre profissionais, usuários e familiares.

Há consistência interna das dimensões resultantes da análise dos dados, com índices alpha de Cronbach ${ }^{13,14}$ que variaram de desejável $(\alpha=0,85)$ a aceitável $(\alpha=0,64)$. 


\section{Discussão}

Como resultado de mais de uma década de pesquisas explorando a interface da bioética e atenção básica, logrou-se construir e validar um instrumento para inventariar problemas éticos na atenção primária à saúde. A aplicação do IPE-APS permite fazer uma 'epidemiologia' ${ }^{15}$ dos problemas éticos na visão dos profissionais de saúde que atuam na atenção primária. Mas, ultrapassa a contabilização porque pode se prestar à mediação da reflexão sobre as questões éticas para a melhoria dos serviços de saúde.

Os resultados do presente estudo validaram o IPE-APS porque mostraram que o instrumento apresenta homogeneidade e consistência interna, com congruência dos itens em cada fator e com o todo ${ }^{16}$. A variância total explicada foi aceitável. Apenas $38,6 \%$ da variância permaneceu inexplorada ${ }^{11}$. Além das medidas estatísticas satisfatórias, houve importante coerência teórica dos fatores resultantes da análise, segundo a literatura revisada ${ }^{12,17}$.

O fator com maior percentual de variância explicada foi 'gestão da atenção primária', pois, independentemente do número de fatores retidos, este sempre se agrupava como o primeiro. Isso provavelmente decorre da importância dessa questão para a APS, especialmente no SUS, no qual se vem propondo que esse nível da atenção seja a porta de entrada do Sistema e, ao mesmo tempo, coordenadora do cuidado pelos diversos pontos da rede de atenção à saúde. Os problemas éticos relacionados à dimensão da gestão da APS guardam relação direta com a ética na gestão dos serviços de saúde, deixando patente a dificuldade de separar a ética dos cuidados de saúde da ética na administração em saúde ${ }^{18}$.

Diversos estudos têm evidenciado a existência de problemas relacionados à gestão do SUS ${ }^{19-21}$. Cada vez mais os serviços de saúde requerem dos municípios o bom desempenho da capacidade de gestão, que há de se voltar para a mudança positiva nos indicadores de saúde da população. Com isso, ganha importância a figura do gestor e de suas decisões político-gerenciais na efetividade e eficiência do SUS. Os profissionais das equipes da ESF e os gestores devem guiar sua prática pelo princípio da responsabilidade ética ${ }^{22}$.

A longitudinalidade do cuidado é o segundo fator do IPE-APS e pode ser entendido como característica central e própria da atenção primária. Para Starfield ${ }^{23}$, longitudinalidade refere-se ao acompanhamento do paciente, ao longo do tempo, por médico generalista ou equipe de saúde, nos múltiplos episódios de doenças e cuidados de promoção e reabilitação da saúde. Para essa continuidade da atenção são imprescindíveis a existência e o reconhecimento de uma fonte regular de cuidados de atenção primária, o estabelecimento de vínculo terapêutico duradouro entre os pacientes e os profissionais de saúde da equipe local e a continuidade informacional ${ }^{24}$. Nesses pontos concentram-se, também, os principais problemas éticos relacionados à longitudinalidade.

O terceiro fator, 'prática das equipes', corrobora estudos que apontaram deficiências na responsabilidade coletiva no trabalho de equipe na ESF, pois os profissionais fragmentam em atuações isoladas o que deveria ser um saber coletivamente construído ${ }^{25}$. Para se alcançar os objetivos da ESF é necessário o efetivo trabalho em equipe, com todos atuando em prol do mesmo objetivo. Nas equipes de saúde, cada profissional exerce sua profissão no bojo de um trabalho coletivo, cujo resultado final depende da contribuição das diferentes áreas do saber à assistência ${ }^{12}$.

o 'perfil profissional', quarto fator, vem ao encontro das evidências de estudos com gestores e trabalhadores do SUS, das distintas esferas de governo, ao mostrarem que, da mesma forma que o desempenho e a gestão dos recursos humanos, a formação dos profissionais afeta, profundamente, a qualidade dos serviços e o grau de satisfação dos usuários ${ }^{26,27}$. O setor público tem dificuldade para contratar profissionais com perfil adequado ao que se pretende e se espera para a APS. No processo de formação dos profissionais, apesar dos recentes esforços de mudança, especialmente com a expansão das horas de estágio na saúde da família, persiste o distanciamento em relação às demandas e necessidades do SUS, com dificuldades para a integração de conhecimentos clínicos e da saúde coletiva ${ }^{12}$.

O fator 'privacidade' revela características próprias da APS, pois a casa dos usuários torna-se extensão do consultório. A relação deixa de ser individual (médico-paciente) para se tornar coletiva (equipe de saúde-família), enriquecendo a discussão de um tópico essencial à bioética, a privacidade das pessoas autônomas, com os problemas específicos e peculiares deste nível da atenção. A privacidade é um princípio derivado da autonomia e engloba a intimidade, a vida privada e a honra das pessoas. No trabalho em equipe multiprofissional, a troca de informações é fundamental para a boa qualidade na assistência. Porém, as informações para os membros das equipes hão de se limitar às necessárias para cada um realizar suas atividades em benefício do usuário ${ }^{28}$. 
No sexto fator, 'sigilo profissional', levanta-se que a questão do segredo não está necessariamente vinculada ao contato direto entre informante e ouvinte. Em função do atendimento aos usuários e famílias, a informação pode ser obtida por uma ou mais pessoas e a confidencialidade deveria ser preservada, como respeito a um direito do usuário ${ }^{29}$. Estudo brasileiro, realizado por Fortes e Spinetti ${ }^{30}$, constatou que os agentes comunitários de saúde preocupam-se com o princípio ético da privacidade e com a necessidade de manter o sigilo, como obrigação ética dos profissionais de saúde.

Ressalte-se que os últimos dois fatores guardam semelhanças entre si, pois os termos 'sigilo' e 'privacidade' se confundem e os problemas éticos descritos em ambos os domínios se entrelaçam na assistência, podendo ser diferentemente interpretados pelos profissionais - talvez por isso tenham apresentado os menores índices de alpha de Cronbach $(0,70$ e 0,64 , respectivamente).

De fato, a proposta do SUS de trabalho em equipe para a atenção primária, especialmente com a Estratégia Saúde da Família, traz questões éticas sobre o sigilo e a privacidade que requerem aprofundamento das reflexões para chegarmos a respostas que respeitem a dignidade e autonomia dos usuários dos serviços. Entretanto, talvez a confusão que se manifestou na análise desses itens no instrumento seja reflexo de que usuários e profissionais ainda estão atônitos com a capilaridade proporcionada pela ESF, onde equipes e profissionais penetram na privacidade dos lares, na intimidade da dinâmica familiar.

Estudo de Seoane e Fortes ${ }^{31}$ mostra divergências nas opiniões dos usuários quanto às informações sobre sua saúde e vida familiar que repassam ao agente comunitário de saúde. Alguns cogitam limitar as informações, não confiando ao agente sequer itens referentes a sua doença. Porém, há usuários que afirmam compartilhar tudo o que diz respeito a sua saúde, conscientes de que as informações serão levadas à equipe. Apesar de se perpetuarem na prática cotidiana da atenção primária situações de desrespeito à confidencialidade, as equipes de saúde consideram que a preservação do sigilo e privacidade dos usuários é elemento chave para ofertar um serviço de qualidade e humanizado ${ }^{32}$.

A perplexidade de todos os envolvidos nessa proposta de trabalho coletivo reflete-se na formação dos futuros profissionais. Estudantes de medicina reconhecem que a confidencialidade da informação na sua experiência de aprendizagem na atenção básica é ponto essencial no desenvolvimento do sigilo profissional que deverão observar como médicos, mas reconhecem ter dificuldades para definir as situações nas quais é necessário partilhar informações e quando estas devem ser mantidas em segredo ${ }^{33}$. Claro está que a troca de informações entre usuário e profissional depende diretamente do clima de confiança que perpassa a relação clínica e o acolhimento do usuário no serviço ${ }^{34}$.

Estudo com mulheres portadoras de HIV, em uma região do município de São Paulo, mostrou que elas revelam sua condição para a equipe de saúde da família somente quando estabelecem vínculo e confiança com os profissionais. Ao contrário, quando sentem medo e insegurança devido à alguma atitude dos profissionais ou não confiam que o sigilo sobre sua condição será mantido, não revelam seu diagnóstico à equipe da atenção primária ${ }^{35}$. 0 vínculo, a confiança e acolhimento na relação dos serviços e profissionais com os usuários e famílias são elementos fundamentais para a reflexão e condução das questões éticas sobre sigilo e privacidade.

\section{Considerações finais}

O IPE-APS é um instrumento original e inovador para a proposta de desenvolver pesquisas em bioética com o intuito de aprofundar a reflexão acerca dos desafios éticos na prática profissional cotidiana dos serviços de saúde. Desconhece-se, até o momento, a existência de instrumentos semelhantes, validados para medir o mesmo construto. Por isso, o IPE-APS é importante ferramenta na melhoria da atenção à saúde. Ele facilitará a realização de estudos extensos, com grandes amostras, o que, por sua vez, também contribuirá para seguir na validação do próprio instrumento. A aplicação de um mesmo instrumento validado em diferentes locais facilita a comparação dos achados, desde que respeitadas as questões da adaptação cultural.

A aplicabilidade do IPE-APS mostrou-se possível em diferentes circunstâncias e locais de trabalho da atenção primária, sendo um bom recurso para pesquisadores, profissionais e gestores obterem informações sobre o perfil de problemas éticos na visão das equipes dos serviços, orientando o trabalho de comitês de ética, comissões de bioética e programas de educação continuada para a realidade local.

O IPE-APS é um instrumento elaborado com um construto emanado da realidade do SUS e validado por meio de pesquisas qualitativas e quantitativas que lançaram mão de instrumentais da análise de discurso, análise de conteúdo, psicometria, 
estatística. Teoricamente, ancora-se na bioética deliberativa de Diego Gracia. Após esta última pesquisa de validação do IPE-APS, com a qual chegamos a uma terceira versão do instrumento, concluímos que ele possibilita: traçar perfis confiáveis das questões éticas na APS; desencadear a reflexão das equipes e profissionais para a reformulação de atitudes pessoais e processos de trabalho.

Com o IPE-APS abrem-se caminhos para novas pesquisas na interface da bioética com a atenção primária à saúde. A relevância do instrumento é possibilitar a realização de estudos com amostras representativas de populações profissionais para verificar a potencialidade de generalização dos problemas indicados no IPE-APS. Ademais, o uso de um instrumento validado em diferentes regiões, ou seja, sua aplicação em estudos com múltiplos sítios para coleta de dados, permitirá a comparação dos resul- tados para verificar se há influência de fatores culturais, locais, sociais, de organização dos serviços de atenção primária nos problemas éticos vivenciados e identificados pelas equipes, podendo se aquilatar a interferência desses fatores, em caso positivo.

Esses estudos também poderão indicar se há diferenças locais e regionais significativas no perfil de problemas éticos da atenção primária. Com isso, não se defende a mera descrição dos problemas éticos, mas sim sistematizar o reconhecimento da situação, com a geração de evidências, para direcionar ações de transformação da prática da atenção primária no sentido de torná-la mais ética, humanizada e cidadã. Localizando os pontos frágeis, no sentido ético, da atuação das equipes de atenção primária, poderá se dirigir a formação permanente para os tópicos e questões mais relevantes e frequentes em cada realidade.

Os vários projetos desenvolvidos desde o primeiro estudo em 2000 contaram com financiamento do CNPq, Fapesp e Capes sob a forma de auxílio à pesquisa e bolsas iniciação científica, mestrado, estágio pós-doutoral e produtividade.

\section{Referências}

1. Zoboli ELCP. Bioética e atenção básica: um estudo de ética descritiva com enfermeiros e médicos do Programa Saúde da Família. [tese]. São Paulo: USP; 2003.

2. Gracia D. Ethical case deliberation and decision making. Med Health Care Philos. 2003;6(3):22733.

3. Silva LT, Zoboli ELCP, Borges ALV. Bioética e atenção básica: um estudo exploratório dos problemas éticos vividos por enfermeiros e médicos no PSF. Cogitare Enferm. 2006;11(2):133-42.

4. Zoboli ELCP Relación clínica y problemas éticos en atención primaria, São Paulo, Brasil. Aten Prim. 2010;42(8):406-1.

5. Silva LT, Zoboli ELCP. Problemas éticos na atenção primária: a visão de especialistas e profissionais. Revista Brasileira de Bioética. 2007;3(1):27-39.

6. Silva LT. Construção e validação de um instrumento para mensuração de ocorrência de problema ético na atenção básica. [dissertação]. São Paulo: USP; 2008.

7. Grupo Hospitalar Conceição. Atenção à saúde. [Internet]. (acesso 24 jun. 2012). Disponível: http://www.ghc.com.br/default.asp?idMenu=atencao_saude

8. Dassa C. Analyse multidimensionnelle exploratoire et confirmative. Montreal: Université Montréal; 1999.

9. Pestana MH, Gageiro JG. Análise de dados para ciências sociais: a complementaridade do SPSS. $3^{\mathrm{a}}$ ed. Lisboa: Silabo; 2003.

10. Anderson RE, Tatham RL, Black B. Multivariate data analysis. In: Hair JF, colaborador. $5^{\text {th }}$ ed. New Jersey: Prentice Hall; 1998.

11. Tabachinick BG, Fidell LS. Using multivariate statistics. $4^{\text {th }}$ ed. San Francisco: Allyn and Bacon; 2001.

12. Brasil. Conselho Nacional de Secretários de Saúde. Atenção primária e promoção da saúde. Brasília: Conass; 2011.

13. Cronbach LJ. Coefficient alpha and the internal structure of tests. Psychometrika. 1951;16(3):297334.

14. Cronbach LJ, Meehl P. Construct validity in psychological tests. Psychological Bulletin. 1955;52(4):281-302.

15. Fetters MD, Brody H. The epidemiology of bioethics. J Clin Ethics. 1999;10(2):107-15.

16. Pasquali L. Psicometria: teoria dos testes na psicologia e educação. Petrópolis: Vozes; 2003.

17. Brasil. Portaria $n^{\circ} 2.488$, de 21 de outubro de 2011. Aprova a política nacional de atenção básica, estabelecendo a revisão das diretrizes e normas para organização da atenção básica, para a estratégia de saúde da família (ESF) e o programa de agentes comunitários de saúde (PACS). Brasília; 2011. 
18. Silva JA, Dalmaso ASW. Agente comunitário de saúde: o ser, o saber, o fazer. Rio de Janeiro: Fiocruz; 2002. p. 240.

19. Serra CG, Rodrigues PHA. Avaliação da referência e contrarreferência no programa saúde da família na região metropolitana do Rio de Janeiro (RJ, Brasil). Ciênc. \& Saúde Col. 2010;15(3):3.579-86.

20. Mendes EV. A atenção primária à saúde no SUS. Fortaleza: Escola de Saúde Pública do Ceará; 2002.

21. Paim JS, Teixeira CF. Configuração institucional e gestão do Sistema Único de Saúde: problemas e desafios. Ciênc. \& Saúde Col. 2007;12(Supp):1.819-29.

22. Amorim AG, Souza ECF. Problemas éticos vivenciados por dentistas: dialogando com a bioética para ampliar o olhar sobre o cotidiano da prática profissional. Ciênc. \& Saúde Col. 2010;15(3):86978.

23. Starfield B. Atenção primária: equilíbrio entre necessidades de saúde, serviços e tecnologia. Brasília: Unesco; 2002.

24. Haggerty JL, Reid RJ, Freeman GK, Starfield BH, Adair CE, McKendry R. Continuity of care: a multidisciplinary review. BMJ. 2003;327(7.425):1219-21. DOI: http://dx.doi.org/10.1136/ bmj.327.7425.1219

25. Pedrosa JIS, Teles JBM. Consenso e diferenças em equipes do programa de saúde da família. Rev. Saúde Pública. 2001;35(3):303-11.

26. Nogueira R. Avaliação de tendências e prioridades sobre recursos humanos de saúde. Brasília: Opas; 2002. (Rede Observatório de Recursos Humanos de Saúde).

27. Cotta RMM, Morales MSV, Gonzáles AL, Ricos JAD, Cotta Filho JS. Obstáculos e desafios da saúde pública no Brasil. Rev Hospital Clínicas de Porto Alegre. 2002;22(1):25-32.

28. Fortes PAC, Martins CL. A ética, a humanização e a saúde da família. Rev. Bras. Enferm. 2000;53(n. esp):31-3.

29. Francisconi CF, Goldim JR. Aspectos bioéticos da confidencialidade e privacidade. In: Costa SIF, Oselka G, Garrafa V, coordenadores. Iniciação à bioética. Brasília: Conselho Federal de Medicina; 1998. p. 269-84.

30. Fortes CAP, Spinetti RS. O agente comunitário de saúde e a privacidade das informações dos usuários. Cad. Saúde Pública. 2004;20(5):1.328-33.

31. Seoane AF, Fortes PAC. A percepção do usuário do programa saúde da família sobre a privacidade e a confidencialidade de suas informações. Saúde Soc. 2009;18(1):42-9.

32. Bellenzani R, Mendes RF. Sigilo na atenção em DST/Aids: do consultório aos processos organizacionais. Polis e Psique. 2011;1(3):140-65.

33. Ferreira RC, Silva RF, Zanolli MB, Varga CRR. Relações éticas na atenção básica em saúde: a vivência dos estudantes de medicina. Ciênc. Saúde Coletiva. 2009;14 (1 suppl): 1.533-40.

34. Teixeira RR. $O$ acolhimento num serviço de saúde entendido como uma rede de conversações. In: Pinheiro R, Mattos RA, organizadores. Construção da integralidade: cotidiano, saberes e práticas em saúde. Rio de Janeiro: Abrasco; 2003. p. 89-111.

35. Ferreira FC, Nichiata LYI. Mulheres vivendo com Aids e os profissionais do programa saúde da família: revelando o diagnóstico. Rev. Esc. Enferm. USP. 2008;42(3):483-9.

\section{Participação dos autores}

José Roque Junges: coordenador do estudo em Porto Alegre, que constituiu a última fase de validação do IPE-APS. Elma Lourdes Campos Pavone Zoboli: desenvolveu o estudo original que identificou os problemas éticos que compõem o IPE-APS e orientou as pesquisas que compõem as etapas anteriores de validação do instrumento. Marcos Paschoal Patussi: pesquisador responsável pelo braço quantitativo do estudo de Porto Alegre, para validação final e consistência interna do instrumento. Rafaela Schaefer e Carlise Rigon Della Nora: realizaram os trabalhos de campo, estruturação dos bancos de dados e análise dos dados. Todos os autores contribuíram igualmente para a elaboração e revisão final do artigo.

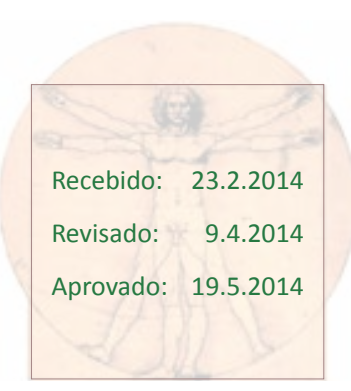




\section{Anexo}

Tabela 1. Fatores, itens, índices de confiabilidade dos fatores e cargas fatoriais dos itens

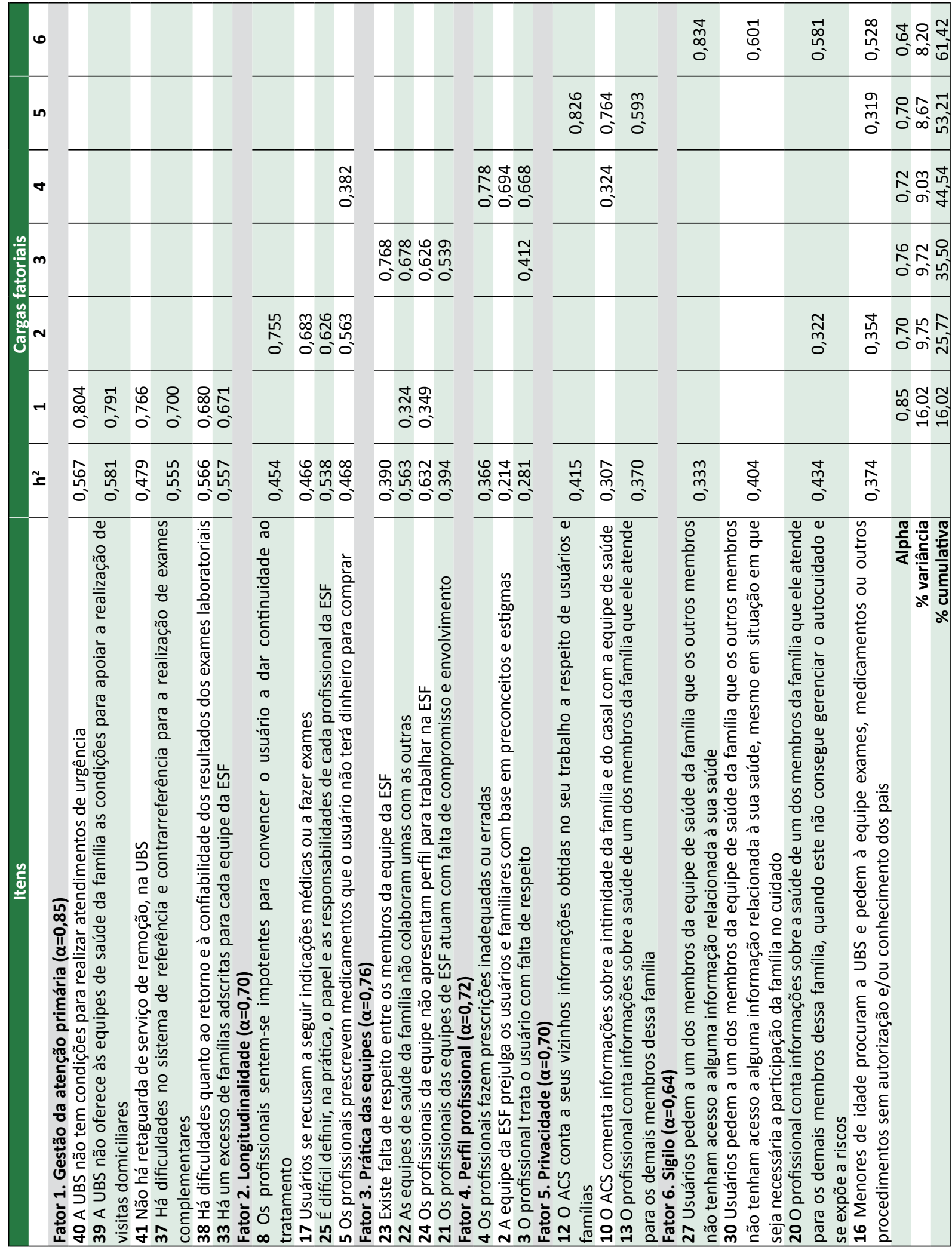

